University of New Hampshire

University of New Hampshire Scholars' Repository

$11-1-2013$

\title{
Science Goals and Overview of the Radiation Belt Storm Probes (RBSP) Energetic Particle, Composition, and Thermal Plasma (ECT) Suite on NASA's Van Allen Probes Mission
}

\author{
Harlan E. Spence \\ University of New Hampshire, harlan.spence@unh.edu \\ G. D. Reeves \\ D. N. Baker \\ J. B. Blake \\ M. Bolton
}

See next page for additional authors

Follow this and additional works at: https://scholars.unh.edu/physics_facpub

Part of the Physics Commons

\section{Recommended Citation}

Spence, Harlan E.; Reeves, G. D.; Baker, D. N.; Blake, J. B.; Bolton, M.; Bourdarie, S.; Chan, A. A.;

Claudepierre, S. G.; Clemmons, J. H.; Cravens, J. P.; Elkington, S. R.; and Fennell, J. F., "Science Goals and Overview of the Radiation Belt Storm Probes (RBSP) Energetic Particle, Composition, and Thermal Plasma (ECT) Suite on NASA's Van Allen Probes Mission" (2013). Space Science Reviews. 144.

https://scholars.unh.edu/physics_facpub/144

This Article is brought to you for free and open access by the Physics at University of New Hampshire Scholars' Repository. It has been accepted for inclusion in Physics Scholarship by an authorized administrator of University of New Hampshire Scholars' Repository. For more information, please contact Scholarly.Communication@unh.edu. 


\section{Authors}

Harlan E. Spence, G. D. Reeves, D. N. Baker, J. B. Blake, M. Bolton, S. Bourdarie, A. A. Chan, S. G.

Claudepierre, J. H. Clemmons, J. P. Cravens, S. R. Elkington, and J. F. Fennell 


\title{
Science Goals and Overview of the Radiation Belt Storm Probes (RBSP) Energetic Particle, Composition, and Thermal Plasma (ECT) Suite on NASA's Van Allen Probes Mission
}

H.E. Spence • G.D. Reeves · D.N. Baker · J.B. Blake • M. Bolton • S. Bourdarie • A.A. Chan - S.G. Claudepierre · J.H. Clemmons · J.P. Cravens · S.R. Elkington • J.F. Fennell • R.H.W. Friedel · H.O. Funsten • J. Goldstein · J.C. Green • A. Guthrie • M.G. Henderson • R.B. Horne • M.K. Hudson • J.-M. Jahn • V.K. Jordanova • S.G. Kanekal • B.W. Klatt • B.A. Larsen · X. Li · E.A. MacDonald • I.R. Mann • J. Niehof • T.P. O'Brien • T.G. Onsager · D. Salvaggio • R.M. Skoug • S.S. Smith • L.L. Suther • M.F. Thomsen · R.M. Thorne

Received: 6 May 2013 / Accepted: 16 July 2013 / Published online: 18 October 2013

(C) The Author(s) 2013. This article is published with open access at Springerlink.com

\begin{abstract}
The Radiation Belt Storm Probes (RBSP)-Energetic Particle, Composition, and Thermal Plasma (ECT) suite contains an innovative complement of particle instruments to ensure the highest quality measurements ever made in the inner magnetosphere and radiation belts. The coordinated RBSP-ECT particle measurements, analyzed in combination with fields and waves observations and state-of-the-art theory and modeling, are necessary for understanding the acceleration, global distribution, and variability of radiation belt elec-
\end{abstract}

H.E. Spence $(\bowtie)$. S.S. Smith

Institute for the Study of Earth, Oceans, and Space, Univ. of New Hampshire, Durham, NH 03824, USA e-mail: Harlan.Spence@unh.edu

G.D. Reeves · R.H.W. Friedel · H.O. Funsten · A. Guthrie · M.G. Henderson · V.K. Jordanova ·

B.A. Larsen · E.A. MacDonald · J. Niehof · R.M. Skoug · M.F. Thomsen

Los Alamos National Laboratory, Los Alamos, NM 87545, USA

D.N. Baker · M. Bolton · S.R. Elkington $\cdot$ X. Li

Laboratory for Atmospheric and Space Physics, University of Colorado, Boulder, CO 90303-7820, USA

J.B. Blake · S.G. Claudepierre · J.H. Clemmons · J.F. Fennell · T.P. O’Brien · D. Salvaggio

The Aerospace Corporation, El Segundo, CA 900009, USA

A.A. Chan

Rice University, Houston, TX 77005-1827, USA

J.P. Cravens

JPC LLC, Port Aransas, TX 78373, USA

J. Goldstein · J.-M. Jahn

Southwest Research Institute, San Antonio, TX 78238-5166, USA

J.C. Green · T.G. Onsager

National Oceanic and Atmospheric Administrations, Boulder, CO 80305, USA 
trons and ions, key science objectives of NASA's Living With a Star program and the Van Allen Probes mission. The RBSP-ECT suite consists of three highly-coordinated instruments: the Magnetic Electron Ion Spectrometer (MagEIS), the Helium Oxygen Proton Electron (HOPE) sensor, and the Relativistic Electron Proton Telescope (REPT). Collectively they cover, continuously, the full electron and ion spectra from one $\mathrm{eV}$ to 10 's of $\mathrm{MeV}$ with sufficient energy resolution, pitch angle coverage and resolution, and with composition measurements in the critical energy range up to $50 \mathrm{keV}$ and also from a few to $50 \mathrm{MeV} /$ nucleon. All three instruments are based on measurement techniques proven in the radiation belts. The instruments use those proven techniques along with innovative new designs, optimized for operation in the most extreme conditions in order to provide unambiguous separation of ions and electrons and clean energy responses even in the presence of extreme penetrating background environments. The design, fabrication and operation of ECT spaceflight instrumentation in the harsh radiation belt environment ensure that particle measurements have the fidelity needed for closure in answering key mission science questions. ECT instrument details are provided in companion papers in this same issue.

In this paper, we describe the science objectives of the RBSP-ECT instrument suite on the Van Allen Probe spacecraft within the context of the overall mission objectives, indicate how the characteristics of the instruments satisfy the requirements to achieve these objectives, provide information about science data collection and dissemination, and conclude with a description of some early mission results.

Keywords Radiation belts - Particle senors - Radiation detection - Space weather · Van Allen Probes

R.B. Horne
British Antarctic Survey, Cambridge CB3 0ET, UK

M.K. Hudson

Dartmouth College, Hanover, NH 03755-1404, USA

S.G. Kanekal

NASA Goddard, Greenbelt, MD 20771, USA

B.W. Klatt

Massachusetts Institute of Technology, Cambridge, MA, USA

I.R. Mann

University of Alberta, Edmonton, AB T6G 2R3, Canada

S. Bourdarie

ONERA-CERT, Toulouse, Cedex 04, France

L.L. Suther

Johns Hopkins University/Applied Physics Laboratory, Laurel, MD, USA

R.M. Thorne

University of California, Los Angeles, 90095, USA

B.W. Klatt

University of Calgary, Alberta, T2N 1N4, Canada 


\section{ECT Science Goals and Objectives}

\subsection{Introduction}

Understanding the acceleration, global distribution, and variability of radiation belt electrons and ions requires a coordinated set of particle measurements analyzed in combination with fields and wave observations and state-of-the-art theory and modeling. The Radiation Belt Storm Probes (RBSP)-Energetic Particle, Composition, and Thermal Plasma (ECT) suite on the Van Allen Probes mission contains a proven complement of particle instruments to ensure the highest quality measurements ever made in the inner magnetosphere.

Instruments in the RBSP-ECT suite were chosen to provide the essential particle measurements needed to achieve science closure on each of eight prime Van Allen Probes mission objectives (see text in Fig. 4 and as outlined below). The suite provides a comprehensive set of particle measurements, optimized to achieve science closure with the fewest instruments and resources. Additionally the RBSP-ECT science team has applied extensive experience in designing, fabricating and operating spaceflight instrumentation for the harsh Van Allen Probes radiation environment.

The HOPE instrument uses an electrostatic top-hat analyzer and time-gated coincidence detectors to measure electrons, protons, helium, and oxygen with energies from $1 \mathrm{eV}$ to $50 \mathrm{keV}$ while rejecting penetrating backgrounds. The MagEIS energetic particle magnetic spectrometer uses magnetic focusing and pulse height analysis to provide the cleanest possible energetic electron measurements over the energy range $20 \mathrm{keV}-4 \mathrm{MeV}$, total ions from $15 \mathrm{keV}$ to $\sim 1 \mathrm{MeV}$, and ion composition from a few to $\sim 50 \mathrm{MeV} /$ nucleon. The REPT telescope covers the challenging electron (proton) energy range above $\sim 2(\sim 8) \mathrm{MeV}$ in order to capture the characteristics of the most intense populations and events using designs adapted from the highly successful SAMPEX mission. These instruments are described respectively in detail in complementary publications of this same special issue (Funsten et al. 2013; Blake et al. 2013; and Baker et al. 2012). The integrated RBSP-ECT suite provides maximum Van Allen Probes science return using a minimum of resources. The RBSP-ECT suite particle measurements are complemented on each Van Allen Probes spacecraft by two other particle sensors which provide additional important information about ion composition in the ring current energy range, RBSPICE (Mitchell et al. 2013), and of the inner zone proton populations, RPS (Mazur et al. 2012).

\subsection{Science Objectives and Context}

The RBSP-ECT Science Team applies measurements from MagEIS, HOPE, and REPT along with theory, models, and auxiliary measurements to answer four fundamental science objectives. These four science objectives consolidate eight prioritized Van Allen Probes mission objectives. These objectives follow naturally from the accumulated scientific understanding developed by earlier missions which motivate the Van Allen Probes mission (please see the introductory section of Baker et al. (2012) for a concise history of those missions and our progress in understanding.) The four Van Allen Probes mission objectives are to:

(1) Determine the physical processes that produce radiation belt enhancement events,

(2) Determine the dominant mechanisms for relativistic electron loss,

(3) Determine how the inner magnetospheric plasma environment controls radiation belt acceleration and loss, and

(4) Develop empirical and physical models for understanding and predicting radiation belt space weather effects 


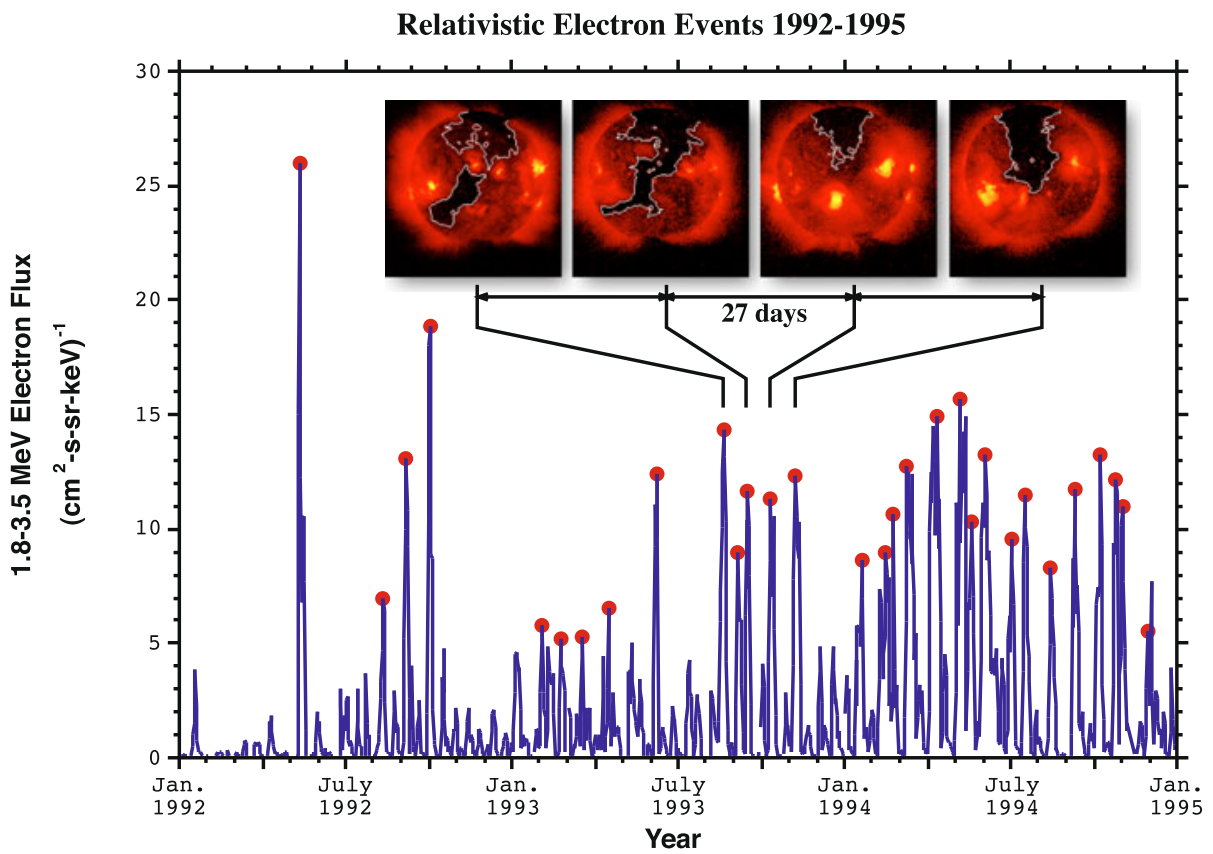

Fig. 1 Daily average geosynchronous electron fluxes 1992-1995 showing quasi-periodic response to high speed solar wind streams from coronal holes (Reeves 1998)

Changes in the Earth's radiation belt environment are primarily caused by two distinct types of solar (interplanetary medium) disturbances: Coronal Mass Ejections (CMEs) and Co-rotating Interaction Regions (CIRs) associated with fast solar wind streams that often originate in solar coronal holes (Fig. 1). Near solar maximum, large geomagnetic storms are frequently initiated by CMEs (Baker et al. 1998). The radiation belt response to CME and non-CME forcing cannot currently be predicted. Storms increase the flux of outer belt electrons only about half the time (Fig. 2). Storms can also decrease relativistic electron fluxes $(\approx 20 \%)$ or change their structure without changing their intensity $(\approx 30 \%)$. This indicates in a given event either acceleration or loss processes can dominate. Acceleration, losses, and adiabatic responses must be understood as a system (Baker et al. 1994). In addition, Blake et al. (1992) have shown that interplanetary shocks can produce 'prompt acceleration' events and combinations of processes under extreme conditions can produce 'slot-filling' events; such observations provide highly-sensitive tests of our physical understanding through theory/model comparisons (e.g. Horne et al. 2005). The RBSP-ECT particle observations will be fully understood only in the context of their connection to the Sun (Mason et al. 2001). We use observations from the LWS Solar Dynamics Observatory (SDO) mission, the ACE satellite, the GOES soft X-ray imagers, and all other relevant available information to fully explore solar and heliospheric sources for all these classes of radiation belt events.

Understanding radiation belt physics requires multi-point measurements of phase space densities. Phase space density is best represented for the radiation belts as a function of the three magnetic invariants—gyro $(\mu$ or $M)$, bounce $(J$ or $K)$, and drift $\left(\Phi\right.$ or $\left.L^{*}\right)$. Particles with the same invariants measured at different locations (therefore different $\mathbf{B}$ ) have different energies and pitch angles. This fact drives the need for continuous spectral and pitch angle 

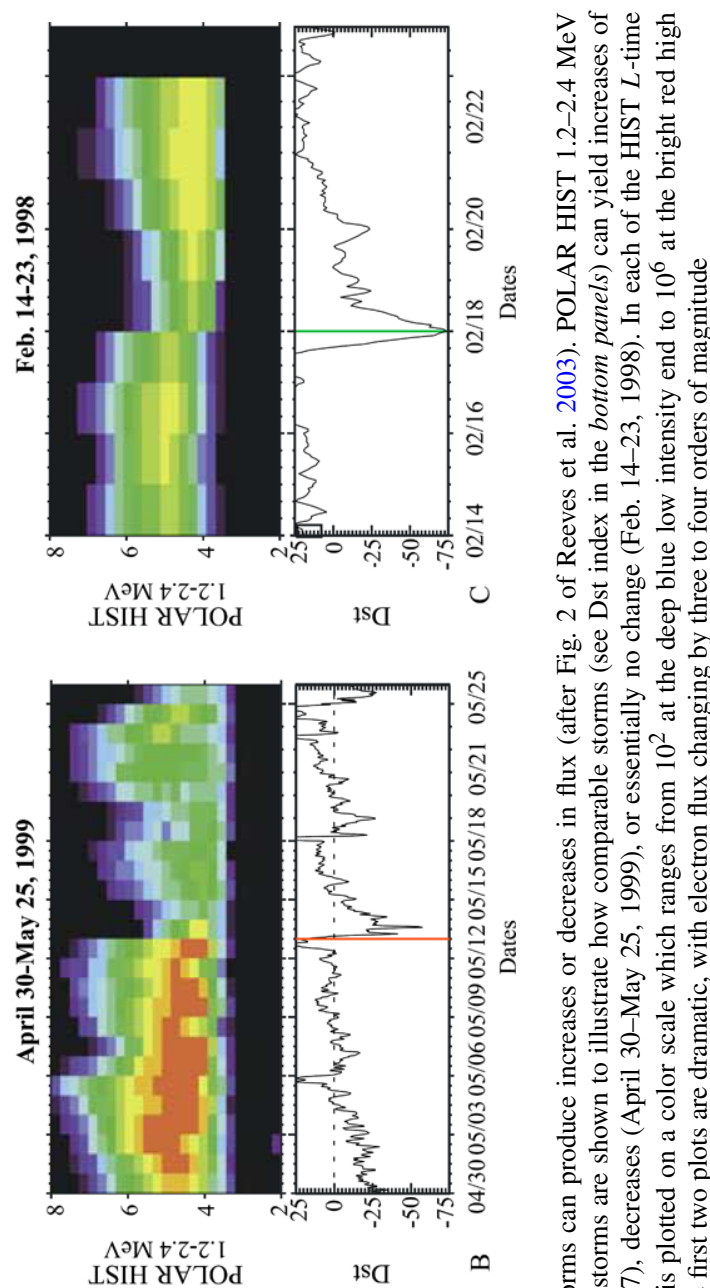

os o v

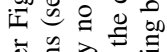

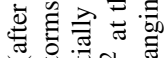

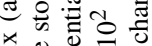

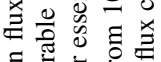

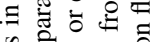

น

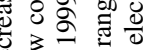

ชู่

䒕交

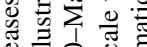

일 웅

으흐흠

\& 运

㐘

‡

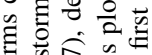

娄会市

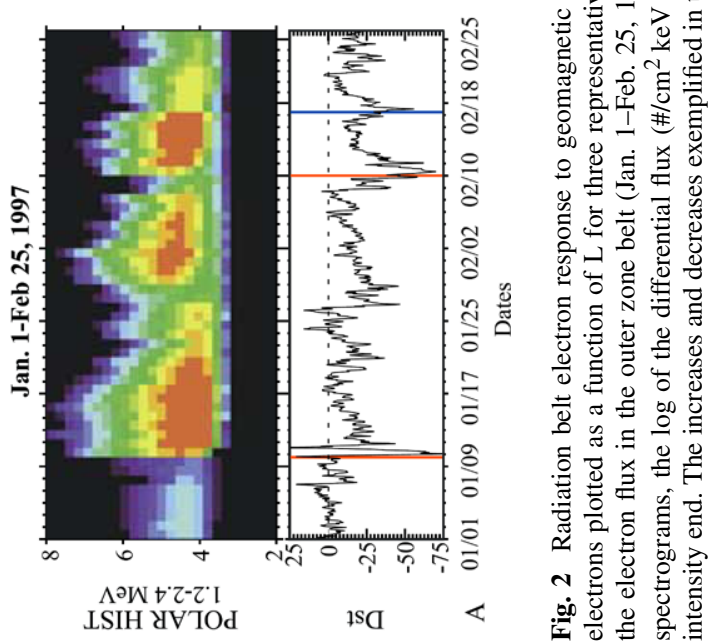



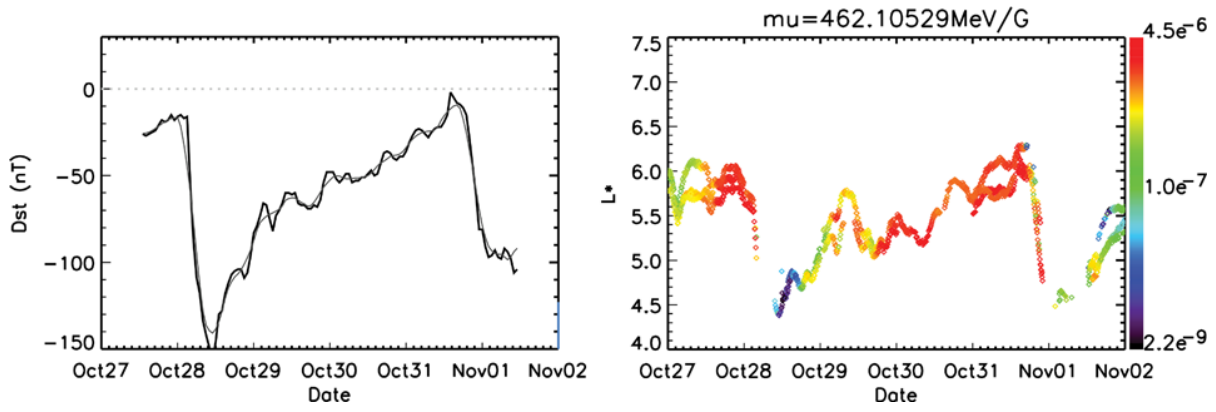

Fig. 3 Dst index and calculated geosynchronous drift shell and phase space densities during a storm using the DREAM model (after Fig. 6 of Reeves et al. 2012). The electron phase space density in units of $(\mathrm{c} / \mathrm{MeV} / \mathrm{cm})^{3}$ is color coded as a function of $L^{*}$ for three geosynchronous satellites (overtraced) during a geomagnetic storm in 2001

coverage with unambiguous instrument energy responses. Temporal and spatial variations will be resolved using the 2-spacecraft Van Allen Probes configuration that produces a full range of radial and azimuthal separations in each local time sector (dawn, dusk, noon, and midnight) over the course of the mission.

The geomagnetic field controls the motion of radiation belt particles. As the field changes during active conditions, large-scale redistribution of radiation belt particles occurs. Recent studies have shown (Reeves et al. 2012), for even a modest storm, that a geosynchronous satellite originally at $L^{*} \approx 6$ will observe particles from $L^{*} \approx 4$ that have moved adiabatically outward during the main phase in response to the inflation of the magnetic field. Figure 3 shows color coded electron phase space density (PSD) as a function of $L^{*}$ for three satellites at geosynchronous orbit during a geomagnetic storm in 2001. As the ring current intensifies (evidenced by the depression in the Dst index) the drift shells "inflate" which produces an "apparent motion" of the satellites to very low $L^{*}$. This adiabatic redistribution (sometimes known as the 'Dst effect') is superposed on any other transport, acceleration, or loss processes.

Non-adiabatic processes come into play when one of the three adiabatic invariants is violated leading to energy diffusion, cross-field transport, and pitch angle scattering (Schulz and Lanzerotti 1974). These processes are produced through the action of convective or inductive electric fields and through wave-particle interactions over a variety of frequencies. Large-scale electric fields transport plasma sheet plasma into the inner magnetosphere where it becomes the source or 'seed' population for radiation belt particles. The characteristics (including composition) of the plasma sheet and plasmasphere determine where and how the waves that control radiation belt dynamics are produced.

The LWS Geospace Mission Definition Team (GMDT) (Kintner et al. 2002) recognized that the storm-time ring current produces the biggest adiabatic changes in the geomagnetic field. Additionally, interaction of the ring current and plasmasphere produces electromagnetic ion-cyclotron (EMIC) waves that strongly interact with outer belt electrons; injected plasma distributions produce broadband chorus across the dawn hemisphere that can both accelerate and precipitate relativistic electrons; and plasmaspheric hiss controls the location and dynamics of the "slot" region. Since the GMDT report was written, however, we have seen considerable advances in our understanding of ring current dynamics. When boundary and initial conditions of these ring current models are well-constrained by measurements of the relevant physical parameters, studies (Jordanova et al. 2001, 2003, 2006; Zaharia et al. 2006) have demonstrated the models' ability to accurately predict the global geomagnetic 
field configuration, as well as predicting VLF wave properties consistent with statistical surveys of wave observations. The RBSP-ECT and RBSPICE particles suites were selected, with these advances in mind, to directly measure those particles and plasma distributions that produce the ring current as well as those properties that determine how, when, and where critical wave populations that accelerate or precipitate relativistic electron are produced.

The RBSP-ECT instrument suite comprising MagEIS, HOPE, and REPT measures the comprehensive spectrum of electrons and ions needed to: calculate adiabatic invariants in storm-time fields; determine dynamic phase space density profiles; understand production of plasma waves; characterize source populations and their transport; and understand the acceleration and loss of radiation belt particles, their space weather effects, and ultimately their response to the variable Sun as well as to processes internal to Earth's magnetosphere.

- Science Objective \#1: Determine the physical processes that produce radiation belt enhancement events

When we consider radiation belt enhancements we distinguish them from "recoveries" following an adiabatic change such as magnetic field stretching or the "Dst effect." True enhancements occur due to the energization of particles when one or more of the magnetic invariants are violated. Betatron/Fermi acceleration (also referred to as "radial" acceleration) occurs when particles are transported from regions of low magnetic field strength to regions of higher magnetic field strength. "Local" acceleration occurs without significant radial transport due to the interaction of particles with local wave populations. "Prompt" acceleration occurs through resonant interaction of a particle's drift motion and the passage of a shock through the magnetosphere. Regardless of type, properties of the resulting enhancement are determined by pre-existing "seed" or source populations as much as by the processes acting on them.

Radiation belt enhancements can occur for both ions and electrons but it is electronsparticularly outer belt and slot electrons - that are the most dynamic. Therefore, while we investigate the electron and ion populations, the primary RBSP-ECT suite emphasis is on understanding electrons. To accomplish this prime suite objective, we will reach closure on four science questions listed below.

Question 1 What processes are responsible for radial transport and acceleration? (Mission priority \#1)

Question 2 Where, when, and how do local acceleration processes produce radiation belt acceleration? (Mission priority \#2)

Question 3 How does 'prompt acceleration' create new radiation belts? (Mission priority \#3)

Question 4 How do plasma 'seed' or source populations influence the characteristics of relativistic electron events? (Mission priority \#5)

ECT Closure on Science Objective \#1, Question 1 Understanding and predicting radial diffusion and relativistic electron acceleration requires: (1) comprehensive and 'clean' particle measurements over a broad range of energies and pitch angles; (2) measurements of the changing radial phase space density gradients; and (3) correlation with local Van Allen Probes and ground-based wave observations, e.g. CanadianArray for Realtime Investigations of Magnetic Activity (CARISMA; Mann et al. 2008), British Antarctic Survey (BAS), and other magnetometer networks). In conjunction with the Van Allen Probes fields and waves observations and the modeling resources available in the community, the ECT particles suite will provide all the necessary information needed to answer this critical science question. 
Multi-spacecraft measurements with broad and continuous differential (integral fluxes will not suffice) energy coverage is key to this problem. To compare electrons with energies from 0.5 to $10 \mathrm{MeV}$ at $L=2$ to electrons at other $L$-shells that have the same first invariant $(\mu)$ it is critical to have continuous differential energy coverage down to at least $20 \mathrm{keV}$. For non-equatorial particles $(J)$ or for removing adiabatic effects $\left(L^{*}\right)$ energies well below $10 \mathrm{keV}$ become important.

In addition to continuous energy coverage, the ECT instruments have been optimized to provide continuous pitch angle coverage for all expected magnetic field conditions (see Blake et al. 2013, for an extensive discussion of instrument design for optimal pitch angle coverage). This is necessary not only to cover a range of second invariants $(J)$ but also to understand how wave-particle interactions alter the electron pitch angle distributions. Radial diffusion preferentially adds perpendicular momentum producing "pancake" distributions with peaks at $90^{\circ}$, whereas wave-particle interactions can produce a wide range of effects depending on the particular wave properties.

Using the DC magnetic field measurements from EMFISIS (Kletzing et al. 2013) with ECT particle fluxes we will achieve science closure by: (1) measuring the temporal evolution of phase space density gradients over a range of adiabatic invariants, (2) examining the evolution of the pitch angle distributions at different drift shells, (3) correlating with ULF field observations, and (4) looking for drift phase-bunching of electrons that resonate with ULF waves.

ECT Closure on Science Objective \#1, Question 2 The most important observations for understanding the role of local acceleration in radiation belt acceleration are radial phase space density distributions. Our previous studies have shown evidence for local peaks but were limited by the range of $L$-shells (e.g. McAdams et al. 2001; Chen et al. 2005) or by high spacecraft latitudes that limited pitch angle coverage (e.g., Selesnick and Blake 1997; Green and Kivelson 2004). The ECT particles suite (plus magnetic field observations) will provide phase space density gradients over the full range of $\mu, J$, and $L^{*}$ values that are crucial to understand belt dynamics. As we have seen, this requires differential energy and pitch angle coverage from a few $\mathrm{keV}$ to $\sim 1 \mathrm{MeV}$. The RBSP-ECT phase space density measurements will enable two types of studies. One uses each spacecraft to measure profiles of the gradient as each traverses $L$-shells in its orbit, at times when we can assume that radial gradients evolve slowly compared to the orbital period. The second technique uses the two spacecraft to obtain instantaneous gradients as well as measurements of the same point separated by the lag time of the trailing satellite. Van Allen Probes mission strategy designed variable separation of the satellites specifically for this purpose-enabling new understanding of acceleration under rapidly changing conditions.

The orbital sweep and instantaneous multi-point measurement strategies are important for understanding and differentiating the spatial and temporal characteristics of changing energy spectra and pitch angle distributions. We correlate those changes with observed wave characteristics, both locally and statistically. Along with wave measurements, understanding where, when, and how, local acceleration occurs requires measuring where and when we observe the characteristic spectral and pitch angle changes discussed earlier.

Over the course of the 2-year Van Allen Probes mission we expect to observe one or two relativistic electron events per month. We will analyze these events in-depth to understand the detailed processes that produce electron acceleration, transport, and loss. Statistical models for the global distribution of all relevant waves, electron spectra, pitch angle distributions, and radial phase space density will be constructed, as a function of MLT, $L$-shell, latitude, and geomagnetic activity. This resource will be made available to develop statistical, physical, empirical, and data assimilation based models. 
ECT Closure on Science Objective \#1, Question 3 ECT instruments are specifically designed to discover how prompt acceleration events create new radiation belts and the most extreme space radiation conditions such as the transformational 24 March 1991 event (Blake et al. 1992) REPT will cover the energy range above what is practical using magnetic spectrometer techniques and will measure the differential energy of electrons from several to $>10 \mathrm{MeV}$ and ions from 10 to $>100 \mathrm{MeV}$. The shape of the energy spectrum (and the existence of theorized upper energy cutoffs) will test acceleration processes.

Ion telescopes in MagEIS use silicon detectors to measure the differential fluxes, energies, and angular distributions of protons from $\sim 50 \mathrm{keV}$ to $\sim 20 \mathrm{MeV}$, and helium and oxygen ions from a few hundred $\mathrm{keV} /$ nuc to a few $\mathrm{MeV} /$ nuc. Since the heavy solar ions are not stripped, and the charge state varies from event to event, the change in composition between the pre-existing seed and the newly trapped ions will be invaluable in understanding the injection process. The drift rate and thus resonance conditions should be charge-state dependent. Although MagEIS does not measure charge state, one can infer the charge state of newly injected helium and oxygen populations by observing the drift resonance periodicity and then comparing these ion populations from event to event and also from pre-event through event to learn about the efficiency of the injection and acceleration processes. ECT will make the best measurements to date of this important part of the distribution and will provide the radial and energy distributions of the pre-existing populations, the largest source of error in numerical models. Together the ECT instrument suite will make the measurements necessary to understand how prompt acceleration works, how it affects electrons and ions, how often it occurs, under what circumstances, and how it combines with other processes to create sudden changes in the space radiation environment. Because of the promptness of these acceleration processes, identical measurements on two spacecraft are critically needed in order to improve both spatial structure and temporal evolution.

ECT Closure on Science Objective \#1, Question 4 Understanding the energy spectra, pitch angle distributions, and spatial/temporal characteristics of the relativistic electron source populations is as important for predictive understanding of the radiation belts as knowing the acceleration, transport, and loss processes. It reinforces the need for continuous, pitch angle-resolved differential energy coverage-particularly at lower energies. This science objective also benefits strongly from the associated observational and modeling tools of the ECT science team and in the community. Coordinated studies with the THEMIS mission (Angelopoulos 2008), at greater distances from the Earth, will allow us to characterize acceleration and transport of plasma sheet electrons into the inner magnetosphere.

- Science Objective \#2: Determine the dominant mechanisms for relativistic electron loss

Relativistic electron losses occur both during strong geomagnetic activity and during quiet periods. The Solar, Anomalous, and Magnetospheric Particle Explorer (SAMPEX) spacecraft (Baker et al. 1993) observations showed that, even during quiet periods, there is a 'drizzle' of electrons into the loss cone that maintains fluxes at low altitude roughly proportional to trapped fluxes (Kanekal et al. 2001). It is, however, dramatic dropouts of relativistic electrons during active times that raise fundamental, unanswered questions. To accomplish the second objective, we will reach closure on two science questions:

Question 1 Where, when, and how are radiation belt electrons scattered into the atmospheric loss cone? (Mission priority \#2)

Question 2 What is the contribution of magnetopause shadowing to relativistic electron loss? (Mission priority \#2) 
ECT Closure on Science Objective \#2, Question 1 Missions such as SAMPEX have clarified how wave-particle interactions are the cause of the pitch angle scattering producing atmospheric precipitation and relativistic electron loss (Baker et al. 2007), but quantitative questions remain. Science closure on this topic relies heavily on correlated studies of Van Allen Probes wave observations with ECT measurements of electron and ion pitch angle distributions, and with other contemporaneously operating missions measuring precipitation at low altitudes ( $\mathrm{Li}$ et al. 2013; Millan et al. 2013; Spence et al. 2012). Using the 2-spacecraft Van Allen Probes observations we will determine the duration of chorus, EMIC, and hiss emissions in a particular region of space and the temporal evolution of the pitch angle distributions as they interact with the waves. Using statistical studies we will determine the spatial and temporal relationships of wave fields and relativistic electron distributions as a function of $L$-shell, MLT, and activity. We will also determine plasma parameters that control wave growth and amplitude to develop predictive understanding of the magnitude and spatial extent of relativistic electron precipitation.

It is important to note that the RBSP-ECT instruments will not resolve the atmospheric loss cone at most $L$-shells. While this has been attempted on other spacecraft missions (e.g. POLAR) it was not a realistic use of Van Allen Probes resources. Regardless of the angular size of the loss cone, the loss cone fluxes are orders of magnitude lower than trapped fluxes. Both populations cannot be credibly measured by the same instrument. Fortunately, science closure in this area does not require resolving the loss cone. It requires measuring pitch angle distributions near the loss cone and the evolution of trapped pitch angle distributions that are the 'smoking gun' for pitch angle diffusion. The RBSP-ECT instruments were designed to measure distributions in the vicinity of the loss cone during quiet conditions and during active times when the field can be stretched into near horizontal orientations.

ECT Closure on Science Objective \#2, Question 2 Because of their $5.8 R_{E}$ apogee, the Van Allen Probes satellites will only encounter the magnetopause when they are observing on the day side during strong magnetopause compressions. However, the Van Allen Probes satellites along with the LANL-GEO, GOES, THEMIS and other satellites can significantly improve our understanding of magnetopause losses. The ECT particle suite will measure the near-equatorial pitch angle distributions that particle drift paths depend upon critically. At the same time advanced numerical models provide global magnetic field configurations for test particle simulations for both magnetopause compressions and storm-time adiabatic changes. Models such as DREAM that use data assimilation and relativistic electron phase space density matching techniques (Chen et al. 2005; Reeves et al. 2012) are particularly powerful tools for combining the pitch angle and energy-resolved measurements from the ECT instruments with geosynchronous observations of magnetopause compressions. Accurate specification of phase space density and radial gradients will allow us to determine loss-related radial diffusion, parallel to studies of acceleration-related radial diffusion. The ECT observations will significantly advance the state-of-the-art specification and prediction of magnetopause losses.

- Science Objective \#3: Determine how the inner magnetospheric plasma environment controls radiation belt transport, acceleration, and loss

We have discussed how the global magnetic field configuration has a direct effect on radiation belt structure. In addition, inner magnetosphere plasmas also affect belt structure and dynamics, even though those effects are often indirect. These lower energy populations are controlled not only by the magnetic field, but their drifts are also significantly controlled by the global convective electric fields which are measured on the Van Allen Probes by the 
Electric Fields and Waves (EFW) instrument (Wygant et al. 2013). Consequently, to quantify the roles of the inner magnetospheric environment to radiation belt particle transport, acceleration, and loss, we also need to understand the processes that control this environment. To accomplish the third objective, we will reach closure on two science questions:

Question 1 How do we characterize/model adiabatic transport of radiation belt particles? (Mission priority \#4)

Question 2 How do the global and local characteristics of plasma sheet and plasmaspheric populations control radiation belt structure, acceleration, and loss? (Mission priority \#6 and 7)

ECT Closure on Science Objective \#3, Question 1 As discussed in the GMDT report and other publications, specification of the storm-time ring current and changes to the global magnetic field are necessary to understand associated radiation belt changes. Ideally this would be done with energetic ion $\left(\mathrm{H}^{+}, \mathrm{He}^{+}, \mathrm{O}^{+}: E>50 \mathrm{keV}\right)$ measurements, plasma ion composition $\left(\mathrm{H}^{+}, \mathrm{He}^{+}, \mathrm{O}^{+}: E<50 \mathrm{keV}\right)$ measurements, energetic neutral atom (ENA) images (Jorgensen et al. 1997; 2001), and self-consistent ring current and magnetic field models. Thermal $(E<50 \mathrm{keV})$ and energetic $(E>50 \mathrm{keV})$ ions in the inner magnetosphere each carry about half the total plasma pressure (Spence et al. 1987); these pressure-bearing ions thereby control the magnetic field configuration. While Van Allen Probes' two-point data will be used to validate or act as input to global models, only models can provide the 3D magnetic field needed to track electron drift orbits.

Comprehensive spectral and pitch angle coverage of electrons and ions provided by RBSP-ECT and by RBSPICE, along with magnetometer measurements on the Van Allen Probes spacecraft, will be used to specify $\mu$ for locally-mirroring particles and as an input into global field models. Models such as DREAM will provide a data-driven, self-consistent, physical model of the storm-time field (Zaharia et al. 2006) and a basis for calculating adiabatic invariants.

We developed/validated a technique to test global fields using 2-satellite measurements similar to those of the Van Allen Probes mission (Chen et al. 2005). This procedure identifies times when non-collocated satellites measure electrons with the same invariants. At these times, phase space densities should be identical. Discrepancies indicate magnetic field errors. Combining detailed measurements, improved magnetic field models, and accuracy tests will produce an unprecedented dataset.

As with the current generation of models we will establish boundary conditions given by the Magnetospheric Plasma Analyzers (MPA) on the LANL geosynchronous satellites (e.g. Thomsen et al. 1998; Jordanova et al. 2003; Blum et al. 2009). Additionally, the HOPE instrument will provide ion composition measurements (at the same energies as MPA) currently unavailable to modelers. The RBSP-ECT science team plans to use global ENA images from the two TWINS satellites (McComas et al. 2009), expected to be operating concurrently with Van Allen Probes.

ECT Closure on Science Objective \#3, Question 2 The RBSP-ECT particles suite, together with the Van Allen Probes fields and waves measurements and inner magnetosphere models, will provide the necessary elements, not only to understand the general interactions that control radiation belt processes, but also to understand and predict the net effect of those processes in a given relativistic electron event. We will measure the plasma distributions that are responsible for the growth and intensity of EMIC, chorus, and other VLF waves. Those measurements include electron phase space density gradients in the vicinity of the loss cone, plasma densities, ring current ion anisotropies, and the flux of $\mathrm{H}^{+}, \mathrm{H}_{\mathrm{E}}^{+}$, and 
$\mathrm{O}^{+}$ions. We will use ECT particle measurements to both drive and validate global models of plasma sheet, ring current, and plasmasphere particles to determine their location and dynamics and therefore the regions where waves will be produced. In combination with the physical understanding gained from investigations of radiation belt acceleration and loss and with the specification of phase space densities as a function of magnetic invariants we will determine where, when, and how inner magnetosphere plasmas and waves control radiation belt structure and dynamics.

- Science Objective \#4: Develop empirical and physical models for understanding and predicting radiation belt space weather effects

To accomplish the fourth objective, which is at the core of overall LWS program objectives, the RBSP-ECT science team will answer the following science question:

Question 1 How do we predict and model spatial, spectral, and temporal characteristics of radiation belt enhancements, over both long and short times? (Mission priority \#8)

ECT Closure on Science Objective \#4, Question 1 Empirical modeling conducted by the RBSP-ECT team will take a stepped approach, recognizing that different levels of model sophistication are required for different applications. While a statistical distribution of peak flux and integrated dose over a satellite orbit may be adequate for some applications, other applications require a more specific parameterization of flux levels at a given location based on solar wind and geomagnetic conditions. We will develop empirical models to provide statistical and probabilistic assessments of flux levels as a function of energy and location (radius, longitude, and latitude). We will also develop models that incorporate current solar wind and magnetospheric conditions, including estimates of wave intensities, diffusion rates, and magnetic field configuration (e.g., Huang et al. 2010a, 2010b). These empirical models will serve as accurate and easily useable tools to further understanding of the radiation belts, as well as provide information for a variety of LWS applications. These efforts will leverage ongoing team modeling strengths including related space weather efforts.

\section{Science Requirements, Traceability, and Instrument Requirements}

In Sect. 1 we identified four overarching RBSP-ECT science objectives, derived from the eight underlying mission priorities that ECT observations will address. Within each objective, we identified one or more science questions that the ECT suite provides closure on, often in concert with other Van Allen Probes measurements, related data sets whether in space or ground-based, and/or with leveraged modeling activities. Within each question, we identified key measurements required to provide closure and discussed how the resultant data analysis answers the science questions.

The RBSP-ECT Instrument Suite Traceability Matrix (see Figs. 4a, 4b) summarizes the Van Allen Probes mission science priorities and their mapping into our ECT measurement goals and science questions, as well as their flow down into science measurement objectives, and finally to instrument requirements. Figure $4 \mathrm{c}$ demonstrates in detail the mapping needed to go from the "science driven measurement requirements" (listed according to physical processes and plasma population) to the minimum-required "science driven instrument requirements."

The science flow "down" (left to right of Fig. 4a) culminates with the minimum instrument requirements necessary and sufficient to achieve RBSP-ECT science goals. As 


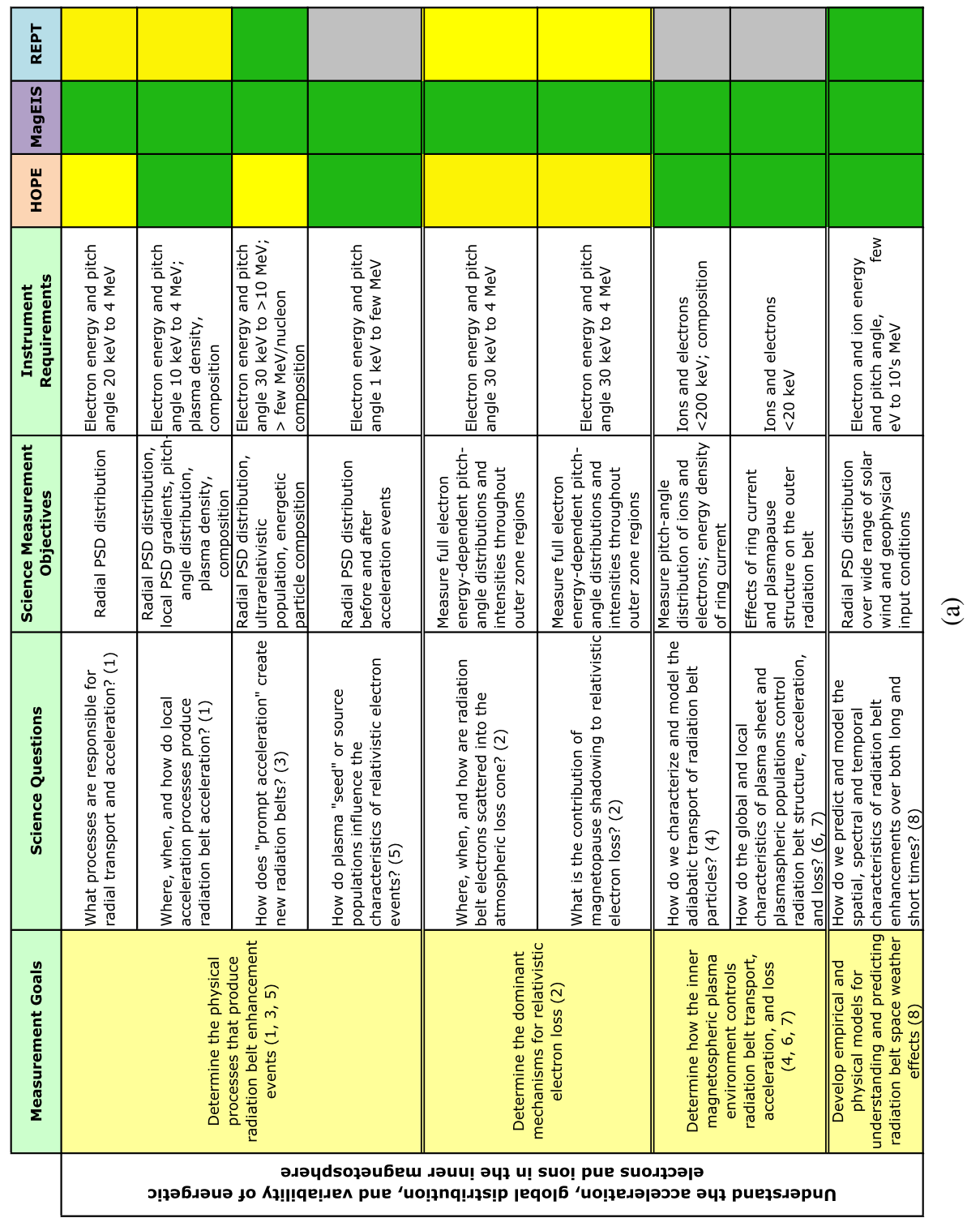




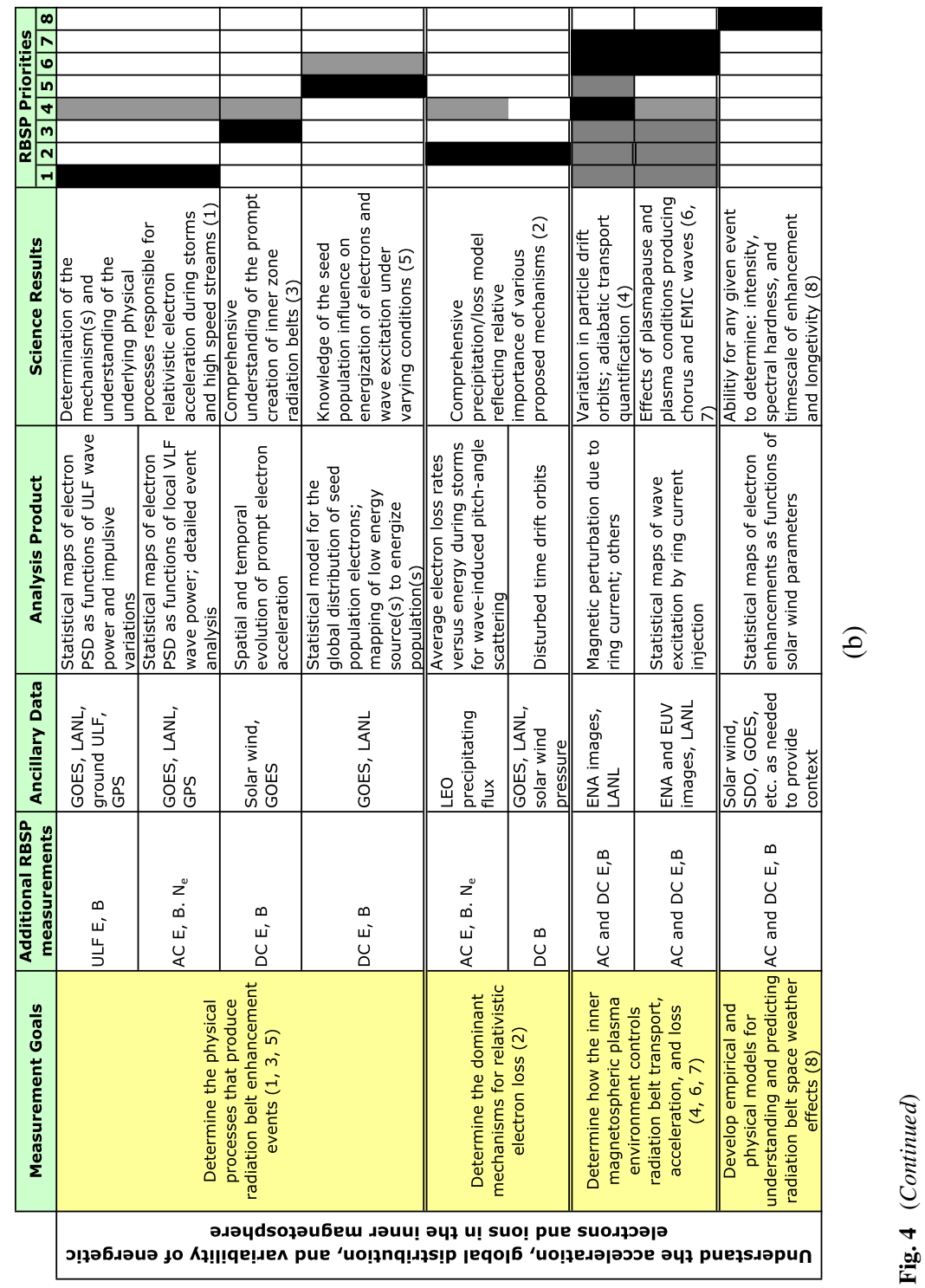




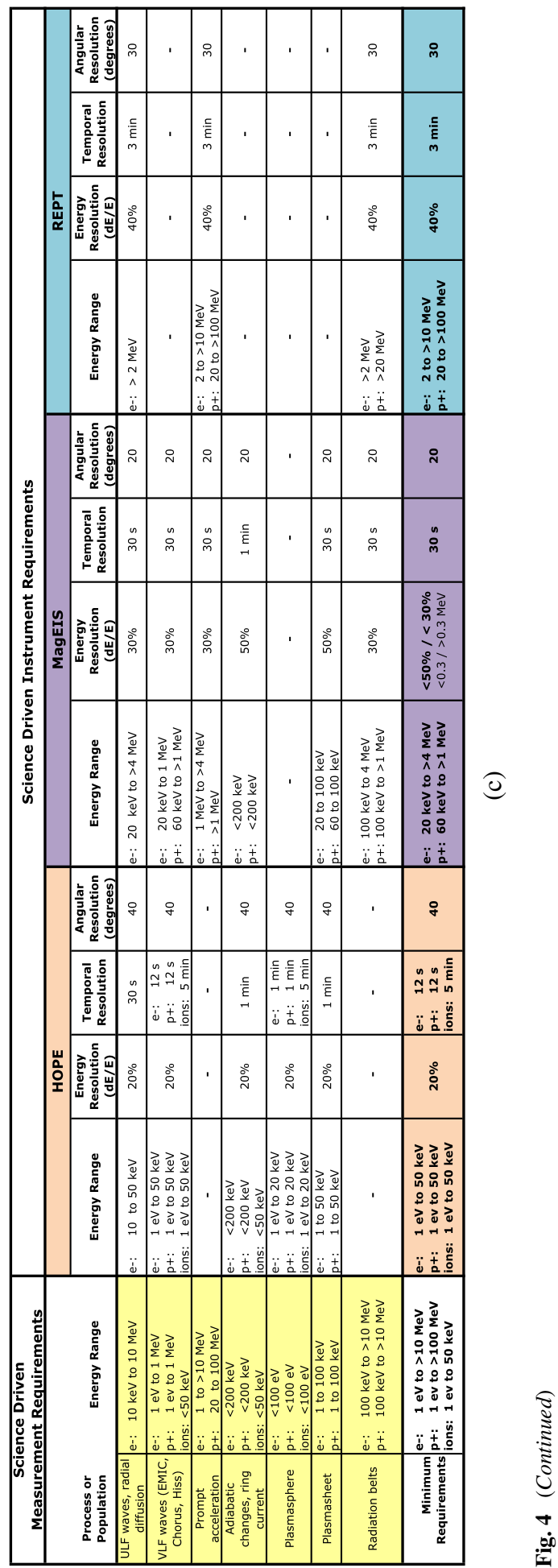




\section{RBSP Prioritized Science Objectives}

1. Differentiating among competing processes affecting the acceleration and transport of radiation belt particles

2. Differentiating among competing processes affecting the precipitation and loss of radiation belt particles

3. Understanding the creation and decay of new radiation belts

4. Quantifying the relative contribution of adiabatic and nonadiabatic processes on energetic particles

5. Understanding the role of "seed" or source populations for relativistic particle events

6. Understanding the effects of rind current and other storm phenomena on radiation belt electrons and ions

7. Understanding how and why the ring current and associated phenomena vary during storms

8. Developing and validating specification models of the radiation belts for solar cycle time scales

Fig. 4 (Continued)

is clearly seen in Fig. 4c, the three ECT instruments (HOPE, MagEIS, and REPT) are designed to meet the critical science-driven instrument requirements in terms of speciesdependent and energy-dependent overall energy ranges, energy resolutions, temporal resolutions, and pitch angle coverages. In the RBSP-ECT companion papers (Funsten et al. 2013; Blake et al. 2013; Baker et al. 2012), we describe all ECT instruments in great detail and show how the instrument requirements are met economically and efficiently as a suite. Color coding in the rightmost columns of Fig. 4a denotes the primary contribution of each instrument to achieving closure on RBSP-ECT science questions; green indicates a required measurement, yellow a desired measurement, and gray indicating that it is not required.

From the leftmost columns working toward the right, the science traceability matrix shows the flow "up" from the instruments back to science closure in Fig. 4b. To answer every RBSP-ECT question, additional Van Allen Probes measurements are identified. For each question, we identify ancillary measurements. We identify next in the matrix the key science analysis product that is responsive to each science question and which flows from the core ECT measurements combined with the other measurements. Finally, we outline the anticipated science result that follows from each analysis product, thereby demonstrating closure back to an initial science question. The final columns of Fig. 4b map the ECT science questions back into the eight Van Allen Probes mission priorities (defined in Fig. 4d). A black bar indicates primary association of the question(s) with the mission priority, gray a secondary association, and white indicating non-applicability.

\subsection{Hope}

The as-flown HOPE instrument performance (see Table 2 of Funsten et al. 2013) meets or exceeds the science measurement requirements developed in the traceability matrix.

The energy range ( 0.001 to $50 \mathrm{keV}$ ) follows directly from the science-measurement traceability matrix. Energy resolution is chosen to adequately resolve all known spectral features in these plasma populations based on previous measurements in the radiation belt, for instance those made by the LANL MPA and Polar HYDRA instruments. The angular coverage and resolution are needed to yield pitch angle distributions with accuracy sufficient to track the pitch angle evolution of plasmasheet electrons and ions (see discussion of Table 2 in Funsten et al. 2013). For plasmasphere ions full spatial coverage is more important than resolution. The time resolution is needed to resolve changes on substorm injection timescales 
for plamasheet electrons; 5 min resolution for the plasma sheet is sufficient to resolve $0.2 R_{E}$ spatial regions. The instrument geometric factors are optimized to cover flux ranges representing the dynamic range of electron and total ion fluxes observed by Polar HYDRA ( $1 \mathrm{eV}$ to $20 \mathrm{keV}$ ) in the region covered by the Van Allen Probes mission.

\subsection{MagEIS}

The as-flown MagEIS instrument performance (see Tables 1 and 2 of Blake et al. 2013) meets or exceeds the science measurement requirements developed in the traceability matrix. The MagEIS instruments cover three different particle populations: ring current electrons $(\sim 20$ to a $\sim 200 \mathrm{keV})$ and radiation belt electrons $(>\sim 200 \mathrm{keV}$ to $>3 \mathrm{MeV})$; ring current protons and radiation beltprotons $(\sim 60 \mathrm{keV}$ to $\sim 20 \mathrm{MeV})$; and energetic helium and oxygen ions from a few hundred $\mathrm{keV} /$ nucleon to a few $\mathrm{MeV} /$ nucleon. We stress that Van Allen Probes science closure will not be possible with instrumentation that lacks differential energy resolution across a broad range of energies. That is especially important in the domain of the relativistic electrons, where some techniques (i.e., solid state telescopes) have not observed important spectral features that only a magnetic spectrometer such as MagEIS can observe. The angular and temporal requirements for MagEIS follow from the same arguments mentioned previously for HOPE.

\subsection{REPT}

REPT provides measurements of the remaining two extreme energy populations needed for closure on ECT science objectives. As with HOPE and MagEIS, the as-flown REPT instrument performance (see Table 1 of Baker et al. 2012) meets or exceeds the science measurement requirements. REPT resolves protons of $\sim 17 \mathrm{MeV}$ to $>100 \mathrm{MeV}$ and electrons between $\sim 1.6 \mathrm{MeV}$ and to $>\sim 19 \mathrm{MeV}$, with a geometric factor optimized for the range of fluxes expected for these dynamic particle populations, and with a time cadence sufficient to resolve dynamical features.

\section{ECT Suite Science Data Collection, Analysis, and Dissemination}

In accordance with the Van Allen Probes mission data policy, the RBSP-ECT instrument team ensures that calibrated high-quality science-grade data, software tools, and related documentation are made available as a public resource to a wide range of end-users including guest investigators (GIs) and members of the space weather community. Our overall plan and timelines for achieving these goals are described in detail below. We anticipate that in many cases science closure on the questions discussed above may be achieved with RBSPECT data in studies led by scientists that are not part of the ECT science team.

\subsection{ECT SOC (Science Operations Center) Overview}

Figure 5 illustrates how the RBSP-ECT data flows from the spacecraft to the various end users. The JHU/APL-run Mission Operations Center (MOC) communicates with the spacecraft and provides command uplink and data downlink. In addition, Near Real Time (NRT) data feeds may also exist as a result of mission-level agreements with external downlink facility operators. The RBSP-ECT Science Operations Center (SOC) is split into two partsthe Command, Telemetry and Ground support part (CTG) and the Science data center part 
Table 1 RBSP-ECT generated data products

\begin{tabular}{|c|c|c|c|}
\hline Data level & Description & Available & Users \\
\hline$L 0$ & $\begin{array}{l}\text { Raw de-commutated telemetry data from } \\
\text { MOC. } L 0 \text { generated by SOC. }\end{array}$ & $\begin{array}{l}\text { Minutes from receipt } \\
\left.\text { (Time }=T_{0}\right)\end{array}$ & SDC, Archives \\
\hline$L 1$ & $\begin{array}{l}L 0+\text { sorted, time-tagged, instrument } \\
\text { separated, units of count rate }\end{array}$ & $T_{0}+<6$ Hours & SDC, ECT team, Archives \\
\hline$L 2$ & $\begin{array}{l}L 1+\text { calibrated and corrected (bkg. } \\
\text { dead-time, etc.), physical units }\end{array}$ & $T_{0}+<2$ weeks & \multirow{3}{*}{$\begin{array}{l}\text { SDC, Co-Is, GIs, US \& Intl } \\
\text { scientific community, } \\
\text { Other LWS Missions, } \\
\text { Archives, Space weather } \\
\text { users, Virtual } \\
\text { Observatories }\end{array}$} \\
\hline$L 3$ & $\begin{array}{l}L 2+\text { B-field derived science products } \\
\text { (pitch angles, moments) }\end{array}$ & $T_{0}+<2$ months & \\
\hline$L 4$ & $\begin{array}{l}L 3+\text { PSD units derived using B-field } \\
\text { models, magnetic coordinates }\end{array}$ & $T_{0}+<1$ year & \\
\hline
\end{tabular}

(SDC). The SOC-CTG transmits instrument commands to the MOC and receives science data, state of health data, housekeeping data and ancillary data (S/C attitude, ephemeris, time offset and scale factor data) from the MOC. Science data would also be received from NRT downlink facilities. The SOC-CTG retrieves and archives these Level-0 ( LO) data products and transmits them together with ancillary data to the SOC-SDC where higher-level products are generated and detailed data analysis, dissemination, and archiving occurs. Since both the SOC-CTG and SOC-SDC reside at Los Alamos National Laboratory (LANL), data processing is highly integrated and a number of resources are effectively and efficiently shared. The RBSP-ECT suite maintains a stripped-down fully redundant emergency SOC capability in a separate facility to ensure reliable failsafe operations.

The SOC-SDC creates higher-level data products (see Table 1) and makes them available for dissemination. Level-1 ( $L 1)$ data products are raw instrument-specific, uncalibrated data files and are archived and made available to the ECT instrument teams. Level-2 data (L2) products (instrument-specific, calibrated, and validated data) are then generated at the SDC with feedback and validation from the suite instrument teams. Higher level analysis of the particle data requires knowledge of the magnetic field vector at each spacecraft and these data are obtained in a preliminary format directly from the EMFISIS Fields and Waves team SOC (quicklook product). Higher quality magnetic field data (EMFISIS level 2) subsequently flows into the SDC when they become available. Level-3 (L3) data are science data products based on these inputs (pitch angles, moments). To enable our science goals, data needs to be folded into adiabatic invariant space with the aid of magnetic field models (Level-4 or L4 products). The ECT SDC will also link to a number of other important and relevant ancillary datasets that will be accessible to the team and all interested users in a timely manner. These include the LANL geosynchronous and GPS particle datasets that are being made accessible as they become available. The team will make available the RBSP-ECT data together with these additional datasets via the SOC-SDC website (http://www.rbsp-ect.lanl.gov).

In addition, the ECT SOC has taken on a mission-wide responsibility of producing a set of value-added ephemeris files, called the "MagEphem" files. These files include the Van Allen Probes satellite position information together with magnetic field model derived coordinates that are useful for radiation belt analysis (such as $L^{*}$ as a function of pitch angle). These files are produced one month ahead based on static field models and orbit predictions, and on dynamic field models once definite orbit files become available. The predict files are also used by the SOC-CTG to plan ahead orbit-specific commanding. All the MagEphem files are provided to the public on the ECT-SOC website. 


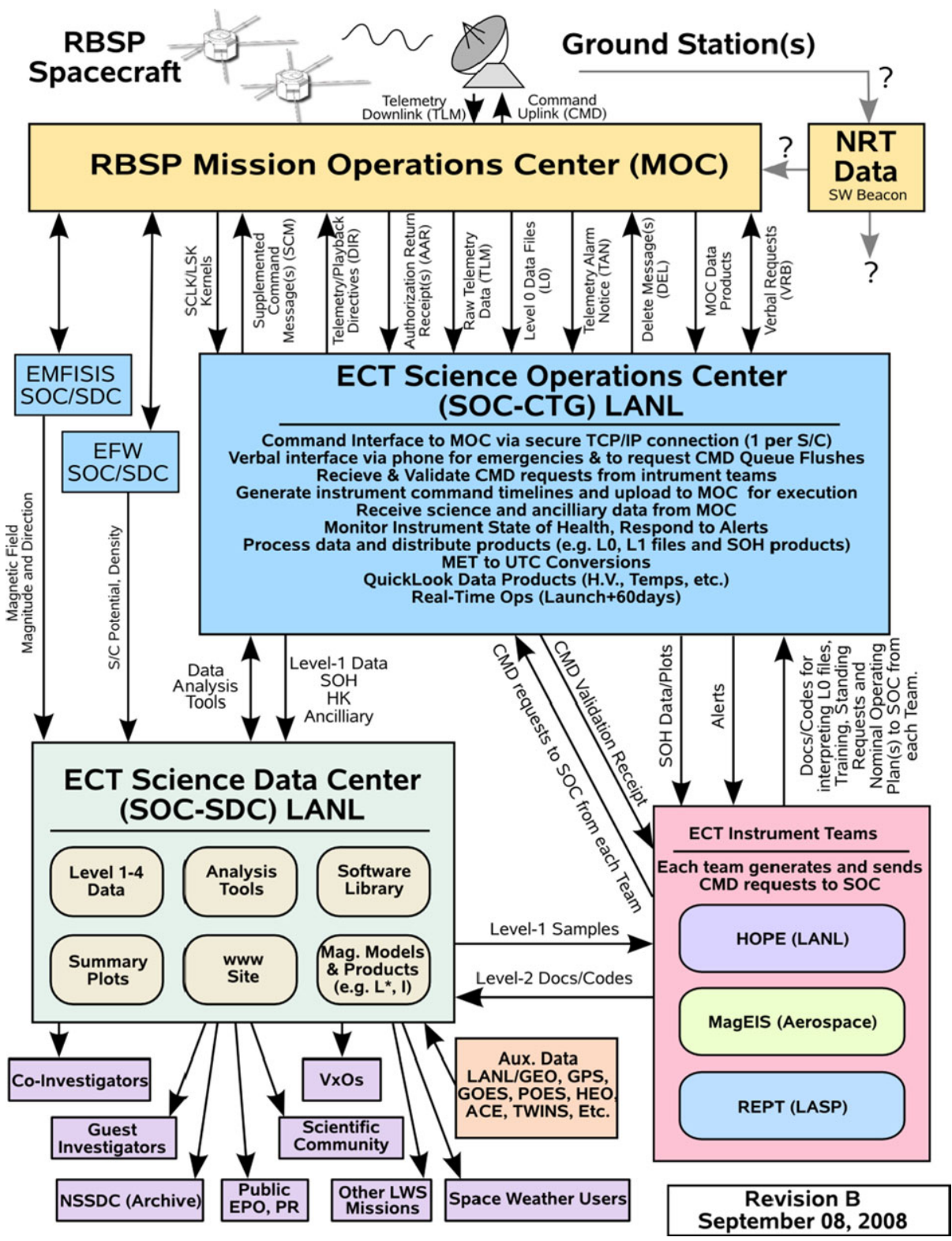

Fig. 5 ECT Data Flow Diagram

The SOC-SDC generates public-domain, science-quality data products available to various end-users in a timely manner: $L 1$ data become available within hours of acquisition from the MOC; $L 2$ data within two weeks; and, $L 3$ data available no later than 2 months. $L 4$ data is available within one year with early Phase-E priority given to intervals selected for community-wide investigation. Expected end-users include: RBSP-ECT co-investigators, Van Allen Probes investigator teams and guest investigators (GIs), the U.S. and interna- 
tional scientific community, virtual observatories, space weather users, archive operators, $\mathrm{E} / \mathrm{PO}$ partners and the public.

\subsection{Science Operations Center Command, Telemetry and Ground Support (SOC-CTG)}

The SOC-CTG operates in two modes—as an automated "Command Scheduler" for normal operations and as a GSEOS (Ground Support Equipment Operating Systems)-based interface for real-time activities. The Command Scheduler provides an easy interface to the three RBSP-ECT suite instrument teams, allowing them to submit command requests simply by email or via a secure SFTP site. The ECT-CTG translates the requests into simple commands, batch commands, or standing requests, uses the predict MagEphem files to schedule orbit specific requests (e.g. "execute at outbound $L=3$ crossing") and manages the execution time requests and the limited on-board command buffers to ensure timely delivery of commands to each spacecraft. In this mode, all commands are time-tagged for a given execution time, uploaded on passes prior to that time and then executed at the target timeallowing commanding for times outside of spacecraft contact times.

The GSEOS interface is used for real-time commanding, only possible during actual spacecraft contact times. This mode was extensively used during instrument commissioning and is used for instrument code uploads and anomaly resolution tasks. The SOC-CTG uses the RBSP-ECT suite instruments team's existing GSEOS implementations from their laboratory development, which provided an integrated system for each team to access their instrument through the single ECT MOC connection provided.

\subsection{Science Operations Center Science Data Center (SOC-SDC)}

The primary function of the RBSP-ECT SOC-SDC is to provide scientific processing, analysis, dissemination, and archiving of the data. It is here that Level-1 and higher data products are generated and where a website exists to facilitate detailed scientific analysis and dissemination of these data. All data are provided in ISTP ( International Solar Terrestrial Physics)-compliant CDF (Common Data Format; Goucher et al. 1994).

$L 0$ science data files and ancillary data files are received from the SOC. $L 1$ data are timesorted and time-tagged in units of count rate. $L 2$ data are corrected (background, dead-time, etc.), calibrated and converted into physically meaningful units and include robust numerical estimates of the statistical uncertainties. ECT data products up to $L 2$ do not require input from other instruments and can therefore be disseminated quickly. Higher-level data products require the addition of magnetic field measurements and model-dependent assumptions. L3 data include pitch-angle sorted data and derived moments. $L 4$ data will be highly validated data converted to phase-space density (PSD) with the aid of realistic global magnetic field models (e.g. Tsyganenko-type empirical models).

The MagEphem processing is also integrated into the SOC-SDC. The MagEphem files are produced using a LANL-developed software library, LANLGEOmag (see Morley et al. 2010), that has been extensively used and validated. This library can produce a large number of parameters and derived calculations utilizing a wide range of common and current magnetic field models, using as input satellite position derived from either three-line elements or a simple text file of $\mathrm{x}, \mathrm{y}, \mathrm{z}$ position data.

The SOC-SDC utilizes so called "QinDenton" files (Qin et al. 2007). These files are produced by the JHU/APL MOC team and contain a near real-time set of useful magnetospheric indices and parameters that are used as input for dynamic magnetic field models such as Tsyganenko and Sitnov (TS04) (Tsyganenko and Sitnov 2005); input parameters 
include measurements such as $L 1$ solar wind properties and geomagnetic indices ( $\mathrm{Kp}$ and Dst). Apart from using these parameters as input to the MagEphem processing, enabling prompt production of MagEphem files with dynamic magnetic field models, the SOC-SDC also makes them available to the broad scientific community through the RBSP-ECT SOC website.

The RBSP-ECT SOC website also provides an on-line repository for the ECT data products, end-user documentation, and data analysis software. High-quality pre-defined summary plots are generated at the SDC and made available via an efficient web-based browsing tool (Autoplot, www.autoplot.org). Software, documentation and data products served to end-users via the SDC are maintained under strict version control for quality control. This is accomplished with a version control system (e.g., Git; Loeliger and McCullough 2012) and definition and adherence to strict file-naming conventions through an advanced database-driven processing chain. Information on the "providence" of each file (the data inputs, software used and their version) is maintained in a hierarchical SQL (Structured Query Language)-lite data base (Date and Darwen 1997), allowing for the full reconstruction of how each version of a given data product was produced. Code updates, data input updates automatically trigger reprocessing and version number increments.

\subsection{Ancillary Data Sets}

These data consist of anticipated coincident inner magnetospheric energetic particle datasets, solar wind data and publicly available ground-based resources (e.g. indices). The SDC provides convenient web-based portals also to these ancillary data.

\subsection{Data Validation}

Primary data validation is the responsibility of the respective RBSP-ECT instrument teams and occurs during the $L 2$ generation phase in the two-week period before public release. All $L 2$ data and plots are marked "preliminary" until checked and cleared by the SOC-SDC lead. Secondary data validation involves the on-orbit cross-calibration of data between the Van Allen Probes spacecraft and auxiliary datasets. Proper inter-calibration of the two spacecraft is required in order to achieve closure on several of the mission scientific objectives (e.g. goals that require determination of PSD gradients).

\subsection{Data Archiving}

The RBSP-ECT SOC archives raw telemetry, $L 0$, and ancillary files. The SDC archive $L 1$ and higher data products. Data, software and documentation generated and stored at the SDC will be prepared for archiving at a site and in a format determined by NASA. LO to $L 3$ datasets will be available for archiving within 2 months of data acquisition and $L 4$ products within 1 year. Routine local archival operations at LANL will include migration of the datasets to a near-line mass storage facility. Following the mission prime phase, an additional year will be used to complete deep-archiving operations. LANL will also provide a long-term resident archive of the full datasets.

\section{Early On-Orbit Performance}

The Van Allen Probes spacecraft launched into their nominal orbits at approximately 4 AM local time on 30 August 2012 from NASA's Kennedy Space Center complex. In order to 


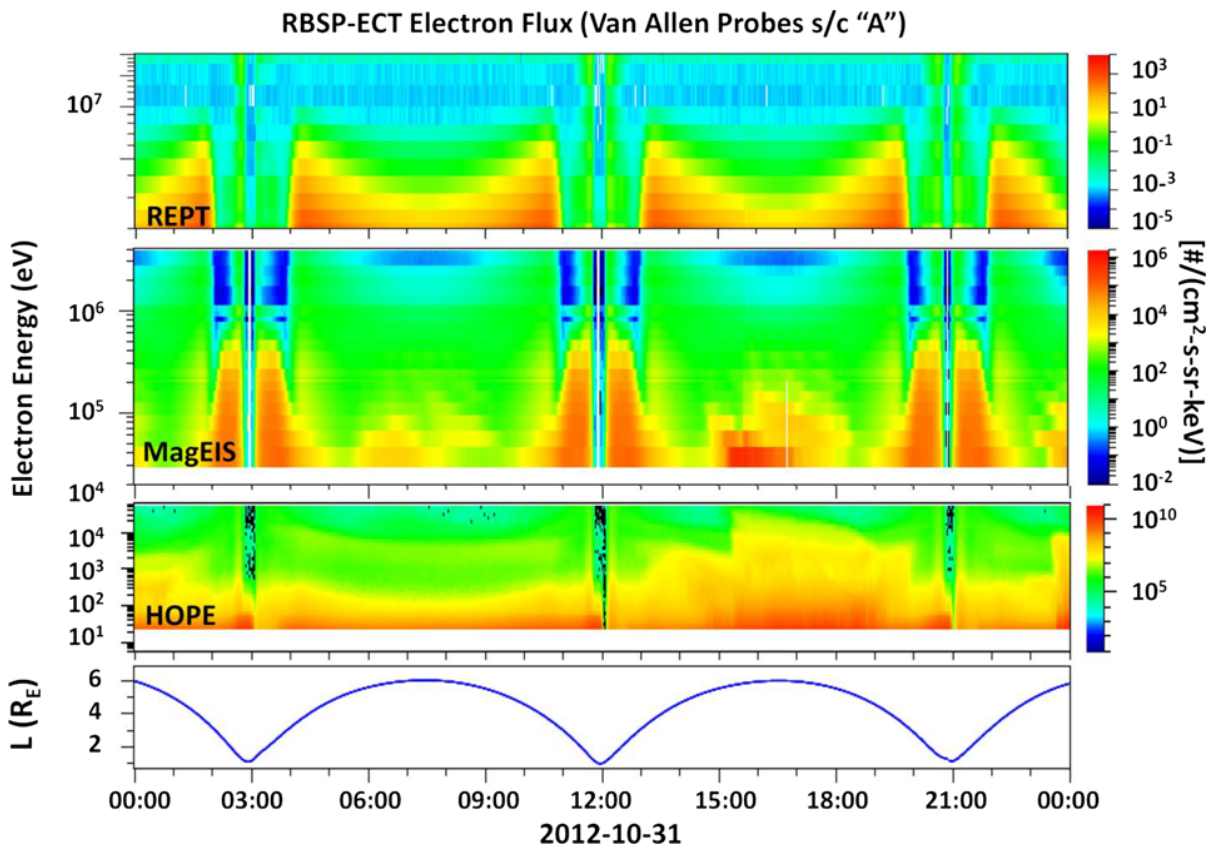

Fig. 6 Early Phase E RBSP-ECT electron energy-time color spectrograms through 2+ orbits on 31 October 2012. Three successive perigee passes of Van Allen Probes spacecraft "A" are seen at 3 UT, 12 UT, and $\sim 21$ UT; intervening apogee traversals occur at $\sim 730$ UT and $\sim 1630$ UT. HOPE covers the lowest electron energies (lower panel), MagEIS the intermediate energies (middle panel), and REPT the highest energies (top panel)

maximize overlap in time with the SAMPEX mission, whose orbit was rapidly deteriorating at that time, the two REPT instruments were turned on early during commissioning on 1 and 2 September 2012. The eight MagEIS instruments were powered on starting on 6 September 2012 and the two HOPE instruments were powered on 3 October 2012. The commissioning of the ECT suite ran smoothly, instrument modes were successfully exercised, look-up tables were assessed and adjusted as necessary, and instrument health was monitored. At the end of the 60-day commissioning, 28 October 2012 which also heralded the beginning of the mission Phase E (science), the ECT suite instrument performance met or exceeded the asdelivered performance, which in turn exceeded all mission measurement requirements.

During subsequent months since the beginning of Phase E, instrument settings have been monitored and adjusted to optimize science performance. A record of such changes is tracked and made available through the RBSP-ECT SOC. Preliminary inflight crosscalibration suggests that the ground-level calibrations are very well understood (as detailed in the individual instrument papers in this special issue). Ongoing cross-calibration continues between HOPE and MagEIS, within the MagEIS units, between MagEIS and REPT, and then across the two Van Allen Probes spacecraft. In addition, important cross-calibrations are also underway with REPT and RPS, RBSPICE with both HOPE and MagEIS, and derived plasma properties of HOPE with derived plasma properties from the fields instruments. These cross-calibrations will continuously improve the quality of our observations, which will be documented by the RBSP-ECT SOC, and will form the basis of several upcoming publications. 
An example of the comprehensive ECT electron energy-time spectrograms from early Phase E operations is shown in Fig. 6. The bottom (middle, top) panel shows electrons as measured by HOPE (MagEIS, REPT) on 31 October 2012, all on Van Allen Probes spacecraft "A". Perigee passes appear as deep reductions in electrons of all energies at $\sim 3$ UT, $\sim 12$ UT, and $\sim 21$ UT. Intervening apogee passages occur at $\sim 630$ and $\sim 1730$ UT. Figure 6 demonstrates several aspects of the comprehensive ECT electron data: outstanding background rejection from the full range of measurements ( $\sim 25 \mathrm{eV}$ to $\sim 10$ 's $\mathrm{MeV})$; overlapping energy coverage by the three instrument types; and with sufficient energy resolution to study the spatial structure and dynamic evolution of electrons throughout the radiation belts, ring current, and plasmasphere. We note that Fig. 6 shows HOPE performance down to $25 \mathrm{eV}$ but the instrument has capability (not shown) down to a few eV (Funsten et al. 2013), the latter being practically limited by spacecraft potential. Similar measurement capabilities (not shown here) span the ion populations, as well. The high quality of the on-orbit ECT charged particle measurements (electrons, protons, and major ions), both in terms of energy and pitch angle, assures the availability of observations needed to reach science closure on the outstanding questions and science objectives discussed above.

In fact, even though the mission is merely months into Phase E, compelling science progress has already occurred using RBSP-ECT observations. Highlights include: the discovery of a dynamic energetic electron "storage ring" (Baker et al. 2013), demonstrating the remarkable capabilities of the RBSP-ECT measurements and the transformational understanding of how the radiations belts can become so exquisitely structured; the related use of theory and complementary observations to demonstrate convincingly that hiss explains the subsequent intensity and pitch angle evolution of these storage ring particles (Thorne et al. 2013); and finally, we have witnessed strong and compelling evidence that a local waveparticle acceleration process led to the prompt recreating of the outer zone electrons that had previously been wiped out by a powerful belt-emptying process (Reeves et al. 2013). These three highlights alone build high confidence that the RBSP-ECT measurements are already fulfilling and will achieve their full promise to answer the key science questions we have posed, as well as lead to exciting and new scientific discoveries that were unimagined before the mission commenced.

Acknowledgements We gratefully acknowledge the Van Allen Probes mission team at the Johns Hopkins University (JHU)/Applied Physics Laboratory (APL) and the Project Science team at JHU/APL, NASA GSFC, and NASA HQ for their invaluable support during the design, development, testing, and early operations of the mission. This work was supported by RBSP-ECT funding provided by JHU/APL Contract No. 967399 under NASA's Prime Contract No. NAS5-01072.

Open Access This article is distributed under the terms of the Creative Commons Attribution License which permits any use, distribution, and reproduction in any medium, provided the original author(s) and the source are credited.

\section{References}

V. Angelopoulos, The THEMIS mission. Space Sci. Rev. (2008). doi:10.1007/s11214-008-9336-1

D.N. Baker, G.M. Mason, O. Figueroa, G. Colon, J. Watzin, R. Aleman, An overview of the SAMPEX mission. IEEE Trans. Geosci. Electron. 31, 531 (1993)

D.N. Baker, J.B. Blake, L.B. Callis, J.R. Cummings, D. Hovestadt, S. Kanekal, B. Klecker, R.A. Mewaldt, R.D. Zwickl, Relativistic electron acceleration and decay time scales in the inner and outer radiation belts. Geophys. Res. Lett. 21, 409 (1994)

D.N. Baker, T. Pulkinnen, X. Li, S.G. Kanekal, J.B. Blake, R.S. Selesnik, M.G. Henderson, G.D. Reeves, H.E. Spence, Coronal mass ejections, magnetic clouds, and relativistic magnetospheric electron events: ISTP. J. Geophys. Res. 103, 17279 (1998) 
D.N. Baker et al., Low-altitude measurements of $2-6 \mathrm{MeV}$ electron trapping lifetimes at $1.5 \leq l \leq 2.5$. Geophys. Res. Lett. 34, L20110 (2007)

D.N. Baker, S.G. Kanekal, V.C. Hoxie, S. Batiste, M. Bolton, X. Li, S.R. Elkington, S. Monk, R. Reukauf, S. Steg, J. Westfall, C. Belting, B. Bolton, D. Braun, B. Cervelli, K. Hubbell, M. Kien, S. Knappmiller, S. Wade, B. Lamprecht, K. Stevens, J. Wallace, A. Yehle, H.E. Spence, R. Friedel, The relativistic electron-proton telescope (REPT) instrument on board the radiation belt storm probes (RBSP) spacecraft: characterization of earth's radiation belt high-energy particle populations. Space Sci. Rev. (2012). doi:10.1007/s11214-012-9950-9

D.N. Baker, S.G. Kanekal, V.C. Hoxie, M.G. Henderson, X. Li, H.E. Spence, S.R. Elkington, R.H. Friedel, J. Goldstein, M.K. Hudson, G.D. Reeves, R.M. Thorne, C.A. Kletzing, S.G. Claudepierre, A longlived relativistic electron storage ring embedded in earth's outer van Allen belt. Science (2013). doi: $10.1126 /$ science. 1233518

J.B. Blake, W.A. Kolasinski, R.W. Fillius, E.G. Mullen, Injection of electrons and protons with energies of tens of MeV into $L<3$ on 24 March 1991. Geophys. Res. Lett. 19, 821 (1992)

J.B. Blake, P.A. Carranza, S.G. Claudepierre, J.H. Clemmons, W.R. Crain, Y. Dotan, J.F. Fennell, F.H. Fuentes, R.M. Galvan, J.S. George, M.G. Henderson, M. Lalic, A.Y. Lin, M.D. Looper, D.J. Mabry, J.E. Mazur, B. McCarthy, C.Q. Nguyen, T.P. O’Brien, M.A. Perez, M.T. Redding, J.L. Roeder, D.J. Salvaggio, G.A. Sorensen, H.E. Spence, S. Yi, M.P. Zakrzewski, The magnetic electron ion spectrometer (MagEIS) instruments aboard the radiation belt storm probes (RBSP) spacecraft. Space Sci. Rev. (2013). doi:10.1007/s11214-013-9991-8

L.W. Blum, E.A. McDonald, S.P. Gary, M.F. Thomsen, H.E. Spence, Ion observations from geosynchronous orbit as a proxy for ion cyclotron wave growth during storm times. J. Geophys. Res. 114 (2009). doi:10.1029/2009JA014396

Y. Chen, R.H.W. Friedel, G.D. Reeves, T.G. Onsager, M.F. Thomsen, Multi-satellite determination of the relativistic electron phase space density at geosynchronous orbit. I. Methodology and initial results during geomagnetic quiet times. J. Geophys. Res. 110, A10210 (2005). doi:10.1029/2004JA010895

C.J. Date, H. Darwen, A Guide to the SQL Standard: a Users Guide to the Standard Database Language $S Q L, 4$ th edn. (Addison Wesley, Reading, 1997). ISBN 978-0-201-96426-4

H.O. Funsten, R.M. Skoug, A.A. Guthrie, J.R. Baldonado, R.W. Harper, K.C. Henderson, K.H. Kihara, J.E. Lake, B.A. Larsen, E.A. MacDonald, A.D. Puckett, V.J. Vigil, R.H. Friedel, M.G. Henderson, J.T. Niehof, G.D. Reeves, M.F. Thomsen, J.-M. Jahn, J. Hanley, D. George, M. Ferris, G. Dunn, M. Freeman, C. Urdiales, A. De Los Santos, C. Nunez, M. Maple, W. Toczynski, T. Taylor, S. Cortinas, E. Edlund, H.E. Spence, J. Cravens, S. Smith, L. Suther (eds.), Helium, Oxygen, Proton, and Electron (HOPE) Mass Spectrometer for the radiation belt storm probes mission. Space Sci. Rev. (2013). doi:10.1007/s11214-013-9968-7

G.W. Goucher, J. Love, H. Leckner, A discipline-independent scientific data management package-the national space science data center's (NSSDC) common data format (CDF), in Proceedings of the 1992 STEP Symposium/5th COSPAR Colloquium, Applied Physics Laboratory, Laurel, Maryland, February (1994)

J.C. Green, M.G. Kivelson, Relativistic electrons in the outer radiation belt: differentiating between acceleration mechanisms. J. Geophys. Res. 109 (2004). doi:10.1029/2003JA010153

R.B. Horne, R.M. Thorne, S.A. Glauert, J.M. Albert, N.P. Meredith, R.R. Anderson, Timescale for radiation belt electron acceleration by whistler mode chorus waves. J. Geophys. Res. 110, A03225 (2005). doi:10.1029/2004JA010811

C.-L. Huang, H.E. Spence, H.J. Singer, W.J. Hughes, Modeling radiation belt radial diffusion in ULF wave fields. 1. Quantifying ULF waves at geosynchronous orbit in data and model. Journal of Geophysical Research 115 (2010a). doi:10.1029/2009JA014917

C.-L. Huang, H.E. Spence, M.K. Hudson, S. Elkington, Modeling radiation belt radial diffusion in ULF wave fields. 2. Estimation of radial diffusion coefficient using combined MHD and particle codes. J. Geophys. Res. (2010b). doi:10.1029/2009JA014918

V.K. Jordanova, C.J. Farrugia, R.M. Thorne, G.V. Khazanov, G.D. Reeves, M.F. Thomsen, Modeling ring current proton precipitation by EMIC waves during the May 14-16, 1997, storm. J. Geophys. Res. 106, 7-22 (2001)

V.K. Jordanova, L.M. Kistler, M.F. Thomsen, C.G. Mouikis, Effects of plasma sheet variability on the fast initial ring current decay. Geophys. Res. Lett. 30(6), 1311 (2003). doi:10.1029/2002GL016576

V.K. Jordanova, Y.S. Miyoshi, S. Zaharia, M.F. Thomsen, G.D. Reeves, D.S. Evans, C.G. Mouikis, J.F. Fennell, Kinetic simulations of ring current evolution during the geospace environment modeling challenge events. J. Geophys. Res. 111, A11S10 (2006). doi:10.1029/2006JA011644

A.M. Jorgensen, H.E. Spence, M.G. Henderson, G.D. Reeves, M. Sugiura, T. Kamei, Global energetic neutral atom (ENA) measurements and their association with the Dst index. Geophys. Res. Lett. 24, 3173 (1997) 
A.M. Jorgensen, M.G. Henderson, E.C. Roelof, G.D. Reeves, H.E. Spence, Charge exchange contribution to the decay of the ring current, measured by energetic neutral atoms. J. Geophys. Res. 106, 1931 (2001)

S.G. Kanekal, D.N. Baker, J.B. Blake, Multisatellite measurement of relativistic electrons: global coherence. J. Geophys. Res. 106, 29721 (2001)

P.M. Kintner, R.S. Lebair, R.A. Hoffman, S. Basu, J.F. Fennell, T.J. Fuller-Rowell, G.A. Germany, G.P. Ginet, M.J. Golightly, R.A. Heelis, M.K. Hudson, R.R. Meier, D.J. Mitchell, R.F. Pfaff, G.D. Reeves, R.M. Robinson, R.W. Shunk, H.J. Singer, J.J. Sojka, R.M. Thorne, R.A. Wolf, J.R. Wygant, W.S. Lewis, The LWS geospace storm investigations: exploring the extremes of space weather, living with a star. Geospace Mission definition team report, NASA/TM-2002-NASA/TM-211613 (2002)

C.A. Kletzing, W.S. Kurth, M. Acuna, R.J. MacDowall, R.B. Torbert, T. Averkamp, D. Bodet, S.R. Bounds, M. Chutter, J. Connerney, D. Crawford, J.S. Dolan, R. Dvorsky, G.B. Hospodarsky, J. Howard, V. Jordanova, R.A. Johnson, D.L. Kirchner, B. Mokrzyki, G. Needell, J. Odom, D. Mark, R. Pfaff Jr., J.R. Phillips, C.W. Piker, S.L. Remington, D. Rowland, O. Santolik, R. Schurr, D. Sheppard, C.W. Smith, R.M. Thorne, J. Tyler, The electric and magnetic field instrument suite and integrated science (EMFISIS) on RBSP. Space Sci. Rev. (2013). doi:10.1007/s11214-013-9993-6

X. Li, S. Palo, R. Kohnert, L. Blum, D. Gerhardt, Q. Schiller, S. Califf, Small mission accomplished by students_-big impact on space weather research. Space Weather J. (2013). doi:10.1002/swe.20025

J. Loeliger, M. McCullough, Version Control with Git, 2nd edn. (O'Reilly, Farnham, 2012). ISBN 978-14493-1638-9

I.R. Mann, D.K. Milling, I.J. Rae, L.G. Ozeke, A. Kale, Z.C. Kale, K.R. Murphy, A. Parent, M. Usanova, D. Pahud, E.-A. Lee, V. Angelopoulos, C.T. Russell, H.J. Singer, The upgraded CARISMA magnetometer array in the THEMIS era. Space Sci. Rev. 141, 413-451 (2008). doi:10.1007/s11214-008-9457-6

G.M. Mason et al., Report on the living with a star (LWS). Science Architecture Team, NASA Headquarters, July 16-18 (2001)

J. Mazur, L. Friesen, A. Lin, D. Mabry, N. Katz, Y. Dotan, J. George, J.B. Blake, M. Looper, M. Redding, T.P. O’Brien, J. Cha, A. Birkitt, P. Carranza, M. Lalic, F. Fuentes, R. Galvan, M. McNab, The relativistic proton spectrometer (RPS) for the radiation belt storm probes mission. Space Sci. Rev. (2012). doi:10.1007/s11214-012-9926-9

K.L. McAdams, G.D. Reeves, R.H.W. Friedel, T.E. Cayton, Multi-satellite comparisons of the radiation belt response to the GEM magnetic storms. J. Geophys. Res. 106, 10869 (2001)

D. McComas, F. Allegrini, J. Baldonado, B. Blake, P. Brandt, J. Burch, J. Clemmons, W. Crain, R. Delapp DeMajistre, D. Everett, H. Fahr, L. Friesen, H. Funsten, J. Goldstein, M. Gruntman, R. Harbaugh, R. Harper, H. Henkel, C. Holmlund, G. Lay, D. Mabry, D. Mitchell, U. Nass, C. Pollock, S. Pope, M. Reno, S. Ritzau, E. Roelof, E. Scime, M. Sivjee, R. Skoug, T.S. Sotirelis, M. Thomsen, C. Urdiales, P. Valek, K. Viherkanto, S. Weidner, T. Ylikorpi, M. Young, J. Zoennchen, The two wide-angle imaging neutral-atom spectrometers (TWINS) NASA mission-of opportunity. Space Sci. Rev. (2009). doi:10.1007/s11214-008-9467-4

R.M. Millan, M.P. McCarthy, J.G. Sample, D.M. Smith, L.D. Thompson, D.G. McGaw, L.A. Woodger, J.G. Hewitt, M.D. Comess, K.B. Yando, A.X. Liang, B.A. Anderson, N.R. Knezek, W.Z. Rexroad, J.M. Scheiman, G.S. Bowers, A.J. Halford, A.B. Collier, M.A. Clilvert, R.P. Lin, M.K. Hudson, The balloon array for RBSP relativistic electron losses (BARREL). Space Sci. Rev. (2013). doi:10.1007/s11214-013-9971-Z

D.G. Mitchell, L.J. Lanzerotti, C.K. Kim, M. Stokes, G. Ho, S. Cooper, A. Ukhorskiy, J.W. Manweiler, J. Jaskulek, D.K. Haggerty, P. Brandt, M. Sitnov, K. Keika, J.R. Hayes, L.E. Brown, R.S. Gurnee, J.C. Hutcheson, K.S. Nelson, N. Paschalidis, E. Rossano, S. Kerem, Radiation belt storm probes ion composition experiment (RBSPICE). Space Sci. Rev. (2013). doi:10.1007/s11214-013-9965-X

S.K. Morley, D.T. Welling, J. Koller, B.A. Larsen, M.G. Henderson, J. Niehof, SpacePy-a python-based library of tools for the space sciences, in Proceedings of the 9th Python in Science Conference (SciPy 2010), ed. by S. van der Walt, J. Millman (2010), pp. 39-45

Z. Qin, R.E. Denton, N.A. Tsyganenko, S. Wolf, Solar wind parameters for magnetospheric magnetic field modeling. Space Weather 5, S11003 (2007). doi:10.1029/2006SW000296

G.D. Reeves, Relativistic electrons and magnetic storms: 1992-1995. Geophys. Res. Lett. 25, 1817 (1998)

G.D. Reeves, K.L. McAdams, R.H.W. Friedel, T.P. O’Brien, Acceleration and loss of relativistic electrons during geomagnetic storms. Geophys. Res. Lett. 30(10), 1529 (2003). doi:10.1029/2002GL016513

G.D. Reeves, Y. Chen, G.S. Cunningham, R.W.H. Friedel, M.G. Henderson, V.K. Jordanova, J. Koller, S.K. Morley, M.F. Thomsen, S. Zaharia, Dynamic radiation environment assimilation model: DREAM. Space Weather 10, S03006 (2012). doi:10.1029/2011sw000729

G.D. Reeves, H.E. Spence, M.G. Henderson, R.H.W. Friedel, H.O. Funsten, D.N. Baker, S.G. Kanekal, J.B. Blake, J.F. Fennell, S.G. Claudepierre, R.M. Thorne, D.L. Turner, C.A. Kletzing, W.S. Kurth, B.A. Larsen, J.T. Niehof, S.K. Morley, Electron acceleration in the heart of the Van Allen radiation belts, Science (submitted and under review) (2013) 
M. Schulz, L.J. Lanzerotti, Particle Diffusion in the Radiation Belts (Springer, New York, 1974)

R.S. Selesnick, J.B. Blake, Dynamics of the outer radiation belt. Geophys. Res. Lett. 24, 1347-1350 (1997)

H.E. Spence, M.G. Kivelson, R.J. Walker, Static magnetic field models consistent with nearly isotropic plasma pressure. Geophys. Res. Lett. 14, 872 (1987)

H.E. Spence, J.B. Blake, A.B. Crew, S. Driscoll, D.M. Klumpar, B.A. Larsen, J. Legere, S. Longworth, E. Mosleh, T.P. O’Brien, S. Smith, L. Springer, M. Widholm, Focusing on size and energy dependence of electron microbursts from the Van Allen radiation belts. Space Weather 10, S11004 (2012). doi:10.1029/2012SW000869

M.F. Thomsen, J.E. Borovsky, D.J. McComas, M.R. Collier, Variability of the ring current source population. Geophys. Res. Lett. 25, 3481-3484 (1998)

R.M. Thorne, W. Li, B. Ni, Q. Ma, J. Bortnik, D.N. Baker, H.E. Spence, G.D. Reeves, M.G. Henderson, C.A. Kletzing, W.S. Kurth, G.B. Hospodarsky, D. Turner, V. Angelopoulos, Evolution and slow decay of an unusual narrow ring of relativistic electrons near $L \sim 3.2$ following the September 2012 magnetic storm. Geophys. Res. Lett. (submitted and under review) (2013)

N.A. Tsyganenko, M.I. Sitnov, Modeling the dynamics of the inner magnetosphere during strong geomagnetic storms. J. Geophys. Res. 110, A03208 (2005). doi:10.1029/2004JA010798

J. Wygant et al., EFW. Space Sci. Rev. (under review) (2013, this issue)

S. Zaharia, V.K. Jordanova, M.F. Thomsen, G.D. Reeves, Self-consistent modeling of magnetic fields and plasmas in the inner magnetosphere: application to a geomagnetic storm. J. Geophys. Res. 111, A11 (2006). doi:10.1029/2006ja011619 